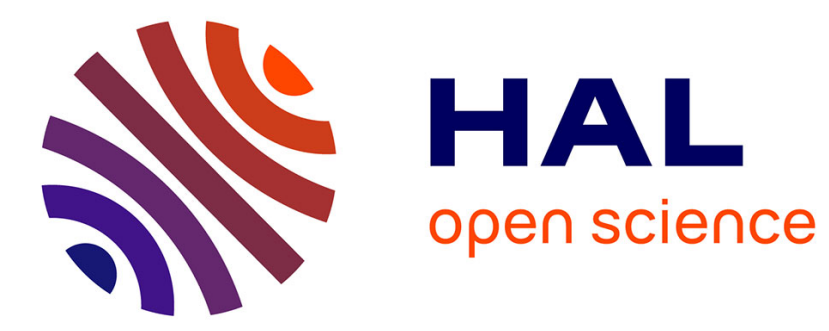

\title{
Brain-stomach coupling: Anatomy, functions, and future avenues of research
}

\author{
Ignacio Rebollo, Nicolai Wolpert, Catherine Tallon-Baudry
}

\section{To cite this version:}

Ignacio Rebollo, Nicolai Wolpert, Catherine Tallon-Baudry. Brain-stomach coupling: Anatomy, functions, and future avenues of research. Current Opinion in Biomedical Engineering, 2021, 18, pp.100270. 10.1016/j.cobme.2021.100270 . hal-03431610

\section{HAL Id: hal-03431610 https://hal.science/hal-03431610}

Submitted on 16 Nov 2021

HAL is a multi-disciplinary open access archive for the deposit and dissemination of scientific research documents, whether they are published or not. The documents may come from teaching and research institutions in France or abroad, or from public or private research centers.
L'archive ouverte pluridisciplinaire HAL, est destinée au dépôt et à la diffusion de documents scientifiques de niveau recherche, publiés ou non, émanant des établissements d'enseignement et de recherche français ou étrangers, des laboratoires publics ou privés. 


\section{Brain-stomach coupling: anatomy,}

\section{functions, and future avenues of research}

Ignacio Rebollo ${ }^{1 *}$, Nicolai Wolpert ${ }^{2}$, Catherine Tallon-Baudry ${ }^{2}$

1-German Institute of Human Nutrition Potsdam-Rehbrücke, Nuthetal, Germany.

2-Laboratoire de Neurosciences Cognitives et Computationnelles, Ecole Normale Supérieure, Inserm U960, PSL University, Paris, France

${ }^{*}$ Corresponding author: Ignacio Rebollo. Email: ignarebo@gmail.com, German Institute of Human Nutrition Potsdam-Rehbrücke, Arthur-Scheunert-Allee 114-116, 14558 Nuthetal, Germany.

Keywords: resting state networks; gastric; gut; electrogastrogram

\section{Highlights}

- A widespread cortical network is coupled to the rhythm of the stomach during rest

- Part of this network might be related to neuromodulation and arousal

- Causal manipulations and novel methods are needed to further understand the gastric network

\section{Abstract}

We have recently discovered the existence in humans of a cortical network synchronized to the gastric rhythm, a constantly generated $0.05 \mathrm{~Hz}$ oscillation that paces the contractions of the stomach necessary for digestion. We present here those recent results, and discuss them in the light of known ascending and descending pathways putatively connecting brain and stomach. We discuss possible functional roles of gastric-brain coupling in homeostasis, arousal and brain function, and review possible causal manipulations of gastric afferents that could be used to test hypotheses about gastric-brain functions beyond rest as well as current methodological limitations. 


\section{1-The gastric network}

Signals from the inside of the body are constantly being relayed to different levels of the central nervous system, which can in turn adjust bodily function to meet actual or expected changes in the internal and external environment $(1,2)$. Signals from within the body, whether consciously perceived or not, can modulate perception, cognition and emotion (3) Here, we focus on the stomach, and review how the continuous stream of ascending gastric signals that reaches the brain, as well as descending autonomic control, contribute to the rich repertoire of intrinsic brain dynamics.

The stomach can be considered as an electrical pacemaker since it creates its own slow rhythm with a frequency around $0.05 \mathrm{~Hz}$ (i.e., 1 cycle every 20 seconds). The muscular layers of the stomach wall contain a specialized type of cell, the Interstitial Cells of Cajal (ICCs), which intrinsically generate and propagate slow pacemaker currents through the ICC network and coupled smooth muscles, making synapse-like connections with afferent fibers of vagal sensory neurons with cell bodies located in the nodose ganglion (Figure 1A). During digestion, these currents set the pace for gastric contractions, but pacemaker activity is present at all times, even outside digestion. While contraction frequency is under the control of ICCs, contraction amplitude is under tight control of the central nervous system. The gastric rhythm can be measured noninvasively using the Electrogastrogram $(4,5)$, i.e. cutaneous electrodes placed on the abdomen (Figure 1B). We have recently proposed (5) a procedure for recording and analyzing EGG data to identify regular gastric rhythms in healthy participants, and applied this approach to recordings in 117 healthy young participants to derive normative distributions of EGG parameters. We observed that while prolonged fasting lead to a more irregular rhythm, neither body mass index, age nor, more unexpectedly, trait anxiety has substantial relationships with EGG amplitude, frequency or regularity.

Given the rhythmic properties of the stomach and the possibility that the gastric rhythm is detected by sensory neurons and relayed up to the neocortex, we hypothesized that it could interact with spontaneous brain activity, even in the absence of conscious perception of gastric activity. We tested whether the gastric rhythm interacts with the resting state networks (RSNs) described using functional magnetic resonance imaging (fMRI), composed of brain regions with correlated activity and complementary functions (6). To test this hypothesis, we recorded fMRI and EGG in healthy human participants at rest, and discovered a widespread network of cortical regions synchronized with the phase of the gastric rhythm (7). The gastric network stands in contrast to classical RSNs in two ways. First, it does not overlap with one RSN, but encompasses portions of different classical RSNs including visual, somatosensory and motor modalities (Figure 1C). Second, the different regions of the gastric network are connected between them and to the stomach with specific, non-zero time delays, as opposed to a classical RSN which is characterized 
by zero-delay connectivity. The method used to identify the gastric network (phase locking value, (8)) does not allow to distinguish between ascending vs. descending regions. However, three arguments indicate a strong contribution of ascending gastric-to-brain neural transmission. First, the gastric rhythm is generated in the stomach even when is decoupled from brain (9). Second, using Magnetoencephalography and time-series causality analysis, we found that the phase of the gastric rhythm modulates the amplitude of the occipital alpha rhythm, the most salient brain rhythm in humans during quiet wakefulness (10). Finally, the electrical stimulation of the rat stomach elicits BOLD and neural responses in the rat brain in a network showing many similarities to the human gastric network, despite known anatomical differences between the two (11-13).

The gastric network appears quite widespread in the brain, encompassing regions that are usually considered to be part of distinct functional ensembles. In the next section, we show how current knowledge on anatomical pathways might account for the spread of the gastric network.

A
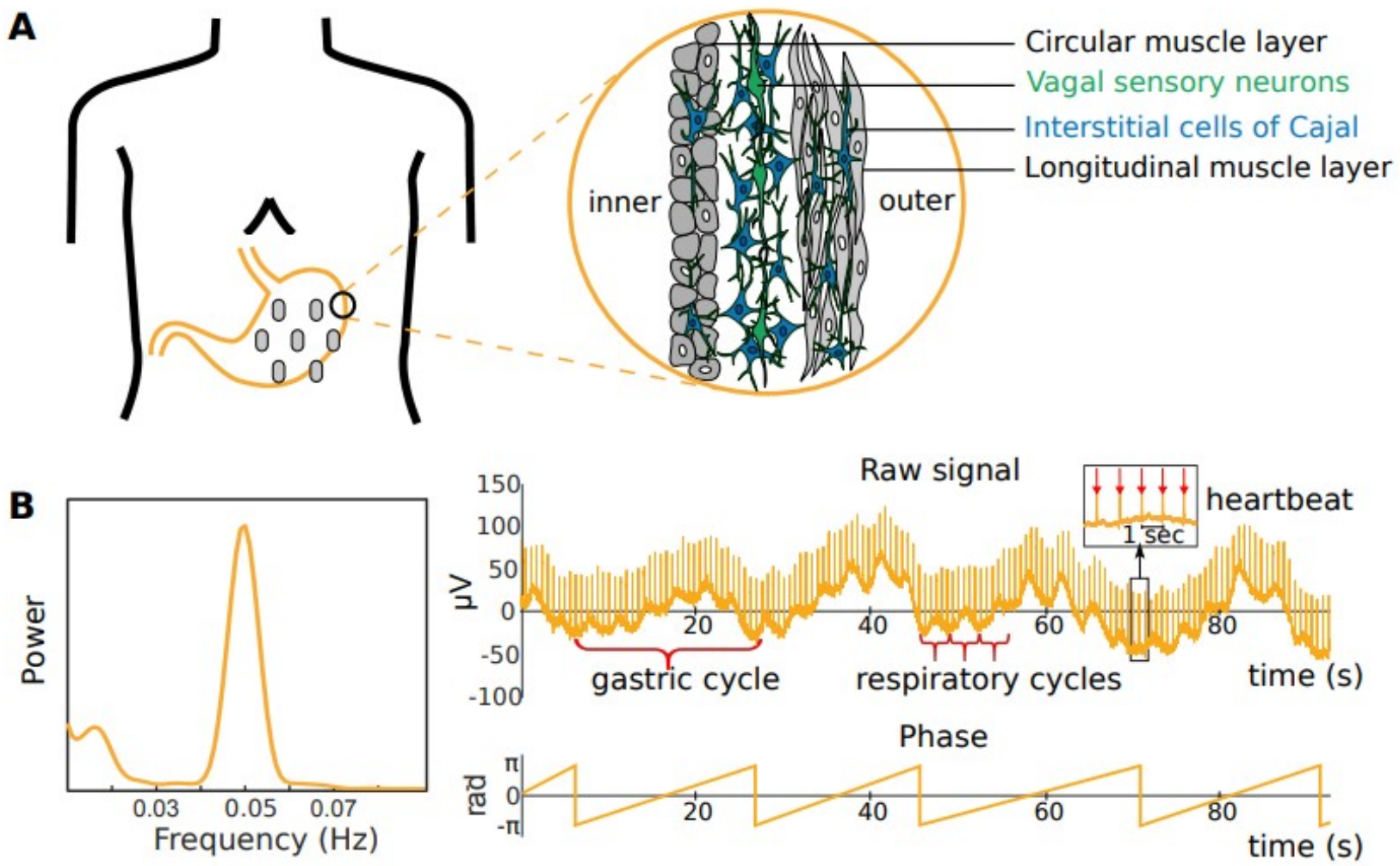

C
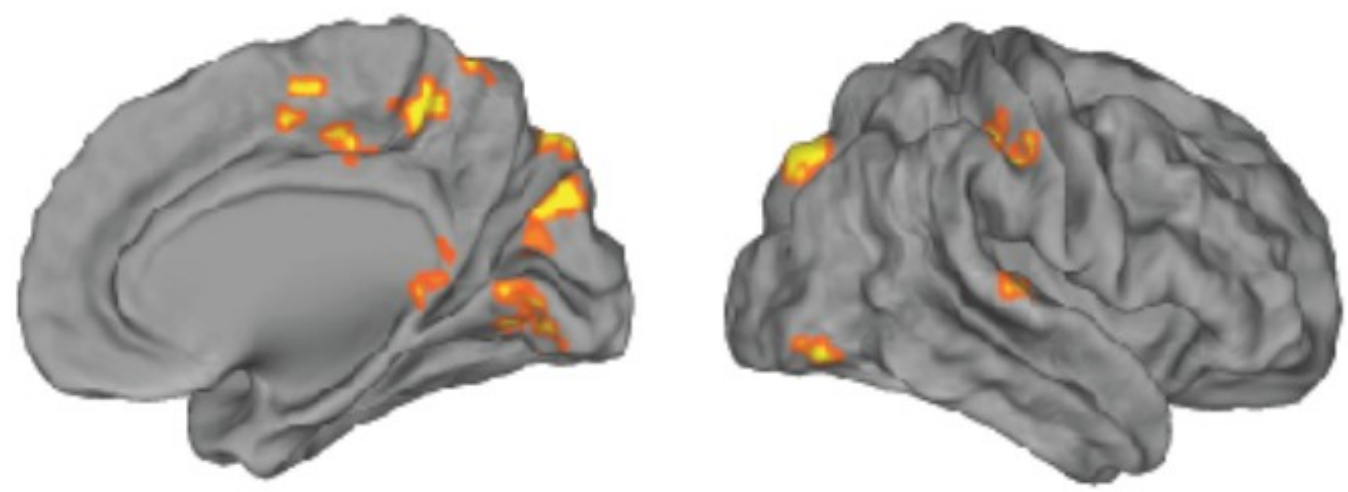
Figure 1: The electrogastrogram and the gastric network. A- The electrogastrogram (EGG) is recorded with cutaneous electrodes placed on the abdomen. The pacemaker cells generating the gastric rhythm, known as the Interstitial Cells of Cajal (ICCs, blue), are located between the circular and longitudinal muscle layers of the stomach wall. Pacemaker currents are passed through the ICC network and passively conducted into coupled muscle cells. ICCs also make synapse-like contact with afferent fibers of vagal sensory neurons (green) that can detect mechanical changes in smooth muscles. B- Left: The power spectrum from an EGG electrode typically shows a sharp peak around $0.05 \mathrm{~Hz}$. Right, upper panel: Example of a raw signal from an EGG electrode. The gastric rhythm is visible as cycles of $\sim 20$ seconds length. Respiratory cycles are much faster (typically 3 to $5 \mathrm{~s}$ length), while heartbeats appear as transients every $\sim 0.8 \mathrm{~s}$ (inset). Lower panel: Instantaneous phase of the gastric rhythm. C- Coupling between gastric phase and fMRI BOLD fluctuations reveals the gastric network. Figure redrawn from $(5,7)$.

\section{2-Anatomical connections between brain and stomach}

How information from the external senses reaches the brain is relatively well understood, even if it is still an active area of research (14). Much less is known about visceral pathways, which seem to have two outstanding characteristics. First, vision, audition and touch all have a single specialized thalamic relay nucleus and a primary cortical sensory region. We argue that for visceral signals this view might not hold. Signals from the viscera stomach have multiple entry points (Fig. 2 A) and target numerous cortical areas, notably through multiple thalamic relays (Fig. 2B). Second, visceral organs have also privileged access to the different neuromodulatory systems (Fig. 2C) as well as to major behavioral and autonomic effectors, providing a continuous stream of interoceptive information that could modulate behavior and cognition (15). We examine these two points in more

A

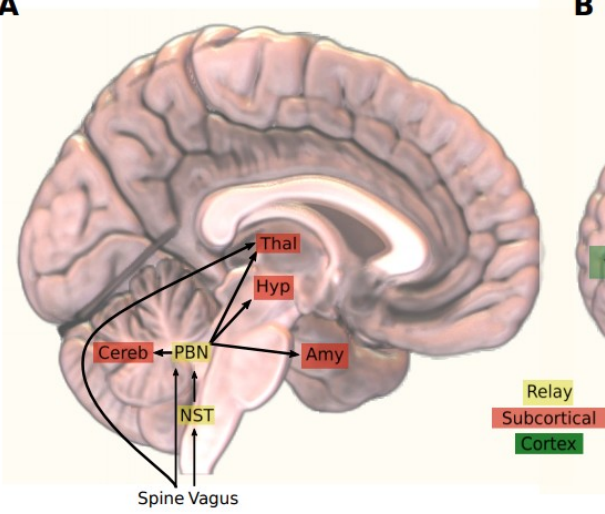

B

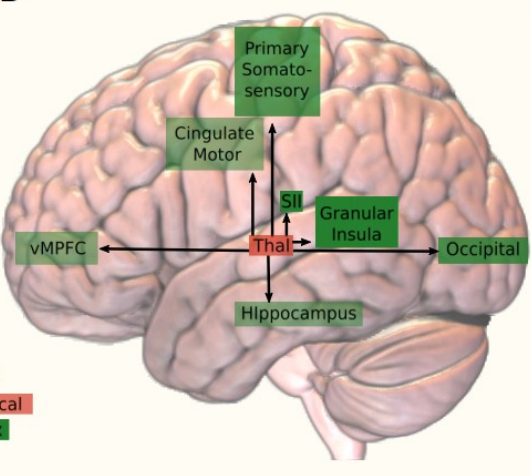

C

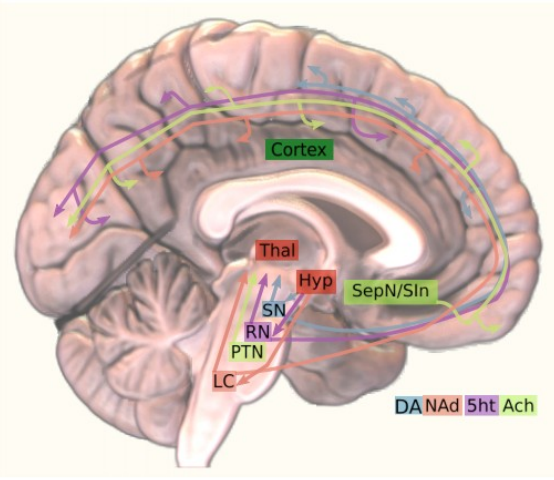

detail below..

Figure 2: Anatomy of ascending stomach projections suggests multiple mechanisms via which gastric signals could influence brain activity A- Overview of entry points of gastric signals in the brain through the vagus nerve and spinal cord. The vagus projects to the Nucleus of the Solitary 
Tract (NST), and together with the spinal cord projects to the Parabrachial Nuclei (PBN). In turn, the PBN provides the majority of inputs to other subcortical structures. Additionally, the spine projects directly to the thalamus via the spinothalamic tract. B- Potential cortical targets of gastric signals, based on functional studies in humans using gastric distention (20), tracing studies of the stomach and other visceral organs in rodents and monkeys $(14,18,22)$ and electrophysiological studies in several species (19). Regions located on the medial wall appear in a lighter shade of green $\mathbf{C}$ - Parabrachial input to neuromodulatory structures could be an additional source for gastric input modulating cortical activity. Gastric afferents reach all major neuromodulatory systems (dopaminergic, noradrenergic, histaminic, cholinergic, and glutamatergic). These neuromodulatory systems receive additional inputs from the orexinergic lateral hypothalamic nuclei, that also receive gastric input and have widespread projections to the rest of the brain. Redrawn based on $(14,23)$.

Abbreviations: 5ht, serotoninergic; Amy, Amygdala ; AP, Area Postrema; Cereb, Cerebellum; DA, Dopaminergic; Glu, Glutamatergic; Hyp, Hypothalamus ; LC, Locus Ceruleus ; Nad, noradrenergic; NST, Nucleus of the Solitary Tract; PBN, parabrachial nuclei; PAG, Periaqueductal grey; PTN, Pontine tegmental nuclei; SII, Secondary Somatosensory cortex ; SepN, Septal Nucleus; SIn, Substantia Innominata; SN, Substantia Nigra; ; Thal, Thalamus ; vMPFC, ventromedial Prefrontal Cortex.

Gastric innervation reaches the brain via two pathways: vagal and spinal (Fig 2A). Vagal neurons project to the nucleus of the solitary tract (NST), the first relay and integration center of visceral signals, and then to the parabrachial nuclei (PBN), which integrates vagal and spinal information. Spinal neurons relay information to the parabrachial as well as directly to the thalamus and cortex via the spinothalamic pathway. In turn, the parabrachial nuclei relay gastric input to multiple subcortical and neuromodulatory structures, including major behavioral, autonomic and endocrine effectors (15), such as the Periaqueductal gray, the bed nuclei of the stria terminalis, the central nucleus of the amygdala and several hypothalamic nuclei, as well as the cerebellum, striatum and thalamus. The mapping of gastric and visceral signals at the level of the thalamus is unclear at best. While there are direct projections from the PBN to frontal and insular cortices in rodents, in primates, PBN projections reaches the cortex exclusively through the thalamus (12). Moreover, there are reports of parabrachial-thalamic projections to unimodal and polymodal thalamic nuclei, including somatosensory (16), interoceptive (17) and visual (18) thalamic relays, but whether these projections originate from the visceral or gustatory portions of the PBN is unknown. Additionally, there are direct spinothalamic projections to somatosensory thalamus (19). Properly establishing the thalamic targets of visceral signals is thus an important unanswered research question.

Regarding the cortex, we still do not know with certainty which regions receive gastric input. Evidence from electrophysiological, neuroimaging and anatomical tracing in other visceral organs suggest that visceral signals reach a large set of cortical regions, including primary and secondary 
somatosensory cortices, cingulate motor regions, primary visual cortex, insula, hippocampus and ventromedial prefrontal cortex $(17,19-23)$. Viral tracers are ideal tools to tackle this question, as the virus can travel anterogradely through several synapses. The examination of post-mortem slices obtained after increasingly long survival times reveals the location of infected neurons through increasingly long pathways. Viral tracing revealed projections from the stomach to the agranular insula and perirhinal cortex (15), with longer survival times needed to detect infections in other cortical regions. Using fMRI we have found many of the expected targets of visceral inputs, including somatosensory, cingulate motor and visual cortex (7), indicating that whole-brain functional imaging is a useful methodology for mapping brain-gut interactions, complementing the intrinsic limitations of anatomical tracing.

A further difference between exteroceptive and visceral signals is that visceral signals seem to have privileged access to neuromodulatory structures (Fig. 2C), even in the absence of conscious visceral sensations. Indeed, gastric-parabrachial inputs reach most neuromodulatory systems, as well as the orexinergic lateral hypothalamic (14), which also regulates neuromodulatory activity (15). Thus, by its anatomical connection to neuromodulatory structures, the stomach has the potential to regulate cortical tone, attention, arousal, learning, and reward, and more generally, the integration and segregation of sensory information in the thalamus and cortex (24). While this interesting possibility is supported by the existence of anatomical connections, functional studies are needed to verify whether the stomach modulates neural activity in parabrachial and neuromodulatory structures. A recent study has provided conclusive evidence for a link between gastric signals and dopamine induced reinforced behavior (25) in mice. Further anatomo-functional tracing from body to brain in rodents is needed, as well as studies using high field mapping in humans, to verify functional links with other neurotransmitter systems.

Descending projections from brain to stomach are also beginning to be better understood. A recent study has provided a comprehensive mapping of cortical and subcortical efferents in the rat (26), and by either cutting the vagus or leaving it intact, has allowed to distinguish between spinal and vagal cortical regions. The sympathetic/spinal system, allegedly involved in the 'fight or flight' response, arises mainly in the trunk representation of the primary motor cortex, and to a lesser extent, in primary somatosensory and secondary motor cortices. Vagal cortical neurons, allegedly involved in 'rest and digest' functions, are located mostly in anterior insula and to a lesser degree in the medial prefrontal cortex, and in the central nucleus of the amygdala. While in humans it is not easy to disentangle ascending and descending regions, it is likely that the restingstate gastric network reflects both ascending and descending influences. 


\section{3-Functions of the gastric network}

What are the functions of the gastric network? Given that the function of the stomach is to store and process food, a link with feeding is expected. Interestingly, a recent study has found links between brain activity in the gastric network and weight loss (27). Across 90 individuals undergoing a weight reduction lifestyle intervention, brain activity at $0.05 \mathrm{~Hz}$ predicted prospective weight loss, with subjects displaying reduced weight loss displaying more power at $0.05 \mathrm{~Hz}$ in the gastric network we identified (7). While this study did not measure gastric activity directly, it provides important clues linking homeostatic regulation of energy balance and brain activity in the gastric network.

Gastric inputs also appear tightly related to arousal and sleep. Increases in Locus Coeruleus activity, associated with increased arousal, follow abrupt changes in stomach activity in anesthetized rats (28), while progressive distension of the small intestine induces sleep in cats (29). This pattern of results might indicate that gradual changes in the small intestine signal satiation and a decrease in arousal, but sudden, potentially painful increases gastric distention leads to sudden increases in arousal. Beyond arousal fluctuations, several reports have linked gastro-intestinal activity to specific sleep-stages $(30,31)$. This goes in line with a recent report of an increase in fMRI activity at $0.05 \mathrm{~Hz}$ during light sleep (32), suggesting the possibility that activity at gastric frequency during sleep might be important for energy homeostasis and the restorative functions of sleep.

Some of the brain regions where gastric-brain coupling was observed both with $\mathrm{FMRI}$ and MEG in humans are quite puzzling: why would the occipital cortex, devoted to vision, be part of the gastric network? Of note, these are not isolated results, since in humans, the visual cortex deactivates when the stomach is artificially distended (21), and in cats, neurons in visual cortices tightly follow the myoelectrical activity of the small intestine during non-REM sleep (31). Several interpretations can be considered. First, the occipital cortex might be actively engaged in homeostatic functions, a hypothesis that would be supported by the direct connections between the (visceral or gustatory) parabrachial nuclei to the visual thalamic relay (18). Second, activity in the occipital cortex might be gated by gastric inputs through neuromodulation, reflecting an overall modulation effect related to arousal. This second hypothesis would fit well with the fact that the amplitude of the alpha rhythm, a brain rhythm associated with relaxed states of wakefulness, is partially determined by the phase of the gastric rhythm (10). Alternatively, changes due to neuromodulation could be related to a more specific process regulating the integration of interoceptive and exteroceptive signals, as well as the allocation of mental resources to the monitoring of the body versus the external environment. Finally, it has been proposed that a distributed representation of visceral inputs across numerous brain regions could be used as a kind of "topological glue", i.e. a common reference point facilitating the alignment of the different 
coordinate systems in which information is encoded in different brain areas (3). This fits well with the observation that the gastric network contains regions displaying topographical maps active when seeing, touching or moving the body, as well as regions involved in transforming between egocentric and allocentric reference frame. Alternatively, gastric-brain coupling could be related to bodily self-consciousness, which has been shown to be modulated by temporal contingencies between interoceptive and exteroceptive signals (33).

Thus, gastric-brain coupling might be related to energy homeostasis and vigilance, with an intriguing role for visual cortices that suggests the possibility that the rhythm of the stomach could influence perception and cognition. Given the anatomical pathways connecting the stomach to the amygdala and to neuromodulatory structures, the stomach could also have an influence on conditioned behavior and affective state, but this hypothesis requires further evidence.

\section{4-Causal manipulations of gastric activity}

Causal manipulations of gastric activity in humans and animals could shed light on the mechanisms and functions of brain-stomach synchrony. Feeding is an ecological manipulation of gastric state that could potentially be used to compare brain-stomach synchrony in fasted versus fed states. Transcutaneous vagal stimulation, or non-invasive stimulation of the vagus nerve is also an interesting lead as it has recently been shown to reduce gastric frequency (34) and increase its amplitude (35), but its effect on gastric-brain coupling is unknown. More invasive manipulations such as mechanical distention of the stomach with inflatable balloons have long been used in combination with functional neuroimaging to map gastric afferents in humans (21), but the stress and discomfort associated with the procedure might bias results. Another causal manipulation of the stomach is the application of magnetic pulses to the abdominal wall, which has been performed in macaques (36) to reveal cortical evoked responses to abdominal stimulations that vary in magnitude in the different stages of the sleep-wake cycle. While such a technique could in theory be applied in humans, twitches in abdominal muscles are to be expected - and might well have occurred in monkeys - which hinders the interpretability of the results.

The most drastic intervention is gastrectomy, a procedure to treat obesity or gastric cancer. Depending on the type of gastrectomy, either a portion, or extremely rarely the totality, of the stomach is removed or bypassed. The integrity of stomach-brain pathways, and remaining pacemaker properties, vary depending on the type of surgery. After sleeve gastrectomy, a procedure which reduces the stomach content to approximately $15 \%$ of its original size, patients with obesity (37) display widespread changes in grey matter, notably in occipital, insular, somatosensory, fusiform, amygdala and hippocampal regions. Since these changes are significantly associated with post-operative weight loss, they reflect a mixture of recovery from obesity and reduced gastric input to the brain. Not that while there have been reports of functional 
connectivity changes following gastrectomy in obese patients (38), notably in default network regions, these results might be artifactual, as gastrectomy-induced changes in BMI are associated with changes in head movement, a major confound in functional connectivity analysis (39). Thus, while gastrectomy could provide important insights on gastric-brain coupling, results must be interpreted carefully, and plasticity, metabolic and artifactual changes need to be considered.

More subtle manipulations of gastric afferents are currently being developed in animal models. The method developed by Cao and colleagues (10) for electrically stimulating the external wall of the stomach is a great example. If combined with the severing of the vagus nerve branch innervating the stomach, this model could be used to determine the influence of vagal and spinal pathways in gastric-brain coupling. This method builds upon Gastric Electrical Stimulation (GES), an invasive therapeutic strategy for the treatment of refractory gastroparesis, i.e. difficulties in gastric emptying. By electrically stimulating the stomach surface with surgically implanted electrodes, this treatments aims to reduce vomiting and nausea symptoms. The effects of GES on human brain activity has been assessed in a single study, which revealed increases in metabolic activity in thalamic and caudate nuclei after chronic GES therapy using Positron Emission Tomography (40). GES has also been used concomitantly with fMRI, revealing evoked responses in occipital cortices and brainstem regions in dogs (41), and the modulation of neural activity in the nucleus of the solitary tract in rats (42). GES thus appears at an interesting manipulation for probing gastric-brain coupling in animal models as well as humans.

\section{5-Back to the gut}

In this article, we have mostly focused on the brain and reduced the stomach to a simple current generator. However, the stomach is a more complex organ. The gastric rhythm consists of propagating waves that travel through the stomach (43), a property that has not yet been considered on the brain imaging side. Additionally, important biological information could be obtained by measuring other gastric parameters beyond electrical activity, such as the $\mathrm{pH}$ of the stomach, or the regulation of gastric distention, contractions and size by the autonomic and central nervous system. Similarly, the influence of other gut organs such as the large and small intestine on intrinsic brain activity have remained largely unexplored (but see (44)). Finally, there would be great potential for ambulatory EGG recordings, a method which is still technically challenging but currently in development (45). Potentially in conjunction with ambulatory EEG, this would allow for prolonged monitoring of gastric activity during daily activity and sleep, and open up exciting opportunities for research with clinical applications. Thus, studying brain-stomach synchrony has opened a wide avenue of research with translational potential that calls for further development of biomedical methods. 


\section{Conflict of interest}

The authors declare no conflict of interest

\section{Funding}

This work was supported by the European Research Council (ERC) under the European Union's Horizon 2020 research and innovation program (grant agreement No 670325, Advanced grant BRAVIUS) and by a senior fellowship of the Canadian Institute For Advance Research (CIFAR) program in Brain, Mind and Consciousness to C.T.-B., as well as by Agence Nationale de la Recherche (ANR-17-EURE-0017). IR was supported by a grant from DIM Cerveau et Pensée and Fondation Bettencourt-Schueller.

\section{References}

1. A. D. Craig, How do you feel? Interoception: the sense of the physiological condition of the body. Nature Reviews Neuroscience 3, 655-666 (2002).

2. C. B. Saper, The Central Autonomic Nervous System: Conscious Visceral Perception and Autonomic Pattern Generation. Annual Review of Neuroscience 25, 433-469 (2002).

3. D. Azzalini, I. Rebollo, C. Tallon-Baudry, Visceral Signals Shape Brain Dynamics and Cognition. Trends Cogn. Sci. (Regul. Ed.) (2019) https:/doi.org/10.1016/j.tics.2019.03.007.

4. K. L. Koch, Robert M. Stern, Handbook of Electrogastrography, Oxford University Press (2004).

5. N. Wolpert, I. Rebollo, C. Tallon Baudry, Electrogastrography for psychophysiological research: Practical considerations, analysis pipeline, and normative data in a large sample. Psychophysiology, e13599 (2020).

- Methological recommendations for the recording of Electrogastrogram in humans and normative values in healthy participants

6. B. T. T. Yeo, et al., The organization of the human cerebral cortex estimated by intrinsic functional connectivity. J. Neurophysiol. 106, 1125-1165 (2011).

7. I. Rebollo, A.-D. Devauchelle, B. Béranger, C. Tallon-Baudry, Stomach-brain synchrony reveals a novel, delayed-connectivity resting-state network in humans. eLife Sciences 7, e33321 (2018).

•• First report of gastric network in humans using resting state fMRI and electrogastrogram

8. J.-P. Lachaux, E. Rodriguez, J. Martinerie, F. J. Varela, others, Measuring phase synchrony in brain signals. Human brain mapping 8, 194-208 (1999).

9. N. Suzuki, C. L. Prosser, V. Dahms, Boundary cells between longitudinal and circular layers: essential for electrical slow waves in cat intestine. American Journal of Physiology - Gastrointestinal and Liver Physiology 250, G287-G294 (1986).

10. C. G. Richter, M. Babo-Rebelo, D. Schwartz, C. Tallon-Baudry, Phase-amplitude coupling at the organism level: The amplitude of spontaneous alpha rhythm fluctuations varies with the phase of the infra-slow gastric basal rhythm. NeuroImage 146, 951-958 (2017). 
- First report that the phase of the gastric rhythm constrains the amplitude of ongoing brain oscillations

11. J. Cao, et al., Gastric stimulation drives fast BOLD responses of neural origin. NeuroImage 197, 200 211 (2019).

•• Causal model of the gastric network using fMRI and electrical stimulation of the stomach in rats

12. T. C. Pritchard, R. B. Hamilton, R. Norgren, Projections of the Parabrachial Nucleus in the Old World Monkey. Experimental Neurology 165, 101-117 (2000).

13. C. Amiez, M. Petrides, Neuroimaging Evidence of the Anatomo-Functional Organization of the Human Cingulate Motor Areas. Cerebral Cortex 24, 563-578 (2014).

14. G.-W. Zhang, et al., A Non-canonical Reticular-Limbic Central Auditory Pathway via Medial Septum Contributes to Fear Conditioning. Neuron 97, 406-417.e4 (2018).

15. L. Rinaman, G. Schwartz, Anterograde Transneuronal Viral Tracing of Central Viscerosensory Pathways in Rats. J. Neurosci. 24, 2782-2786 (2004).

- A detailed anatomical tracing study of subcortical targets of gastric inputs in rats

16. S. J. Coen, A. R. Hobson, Q. Aziz, "Chapter 23 - Processing of Gastrointestinal Sensory Signals in the Brain" in Physiology of the Gastrointestinal Tract (Fifth Edition), L. R. Johnson, et al., Eds. (Academic Press, 2012), pp. 689-702.

17. D. F. Cechetto, C. B. Saper, Evidence for a viscerotopic sensory representation in the cortex and thalamus in the rat. Journal of Comparative Neurology 262, 27-45 (1987).

18. A. Erişir, S. C. Van Horn, S. M. Sherman, Relative numbers of cortical and brainstem inputs to the lateral geniculate nucleus. PNAS 94, 1517-1520 (1997).

19. R. P. Dum, D. J. Levinthal, P. L. Strick, The Spinothalamic System Targets Motor and Sensory Areas in the Cerebral Cortex of Monkeys. J. Neurosci. 29, 14223-14235 (2009).

20. V. E. Amassian, Cortical representation of visceral afferents. J. Neurophysiol. 14, 433-444 (1951).

21. I. Van Oudenhove, et al., Cortical deactivations during gastric fundus distension in health: visceral pain-specific response or attenuation of 'default mode' brain function? A $\mathrm{H}_{2}{ }^{15} \mathrm{O}-\mathrm{PET}$ study. Neurogastroenterology \& Motility 21, 259-271 (2009).

22. M. Castle, E. Comoli, A. Loewy, Autonomic brainstem nuclei are linked to the hippocampus. Neuroscience 134, 657-669 (2005).

23. B. A. Vogt, S. W. Derbyshire, Visceral circuits and cingulate-mediated autonomic functions. Cingulate neurobiology and disease, 219-236 (2009).

24. J. M. Shine, Neuromodulatory Influences on Integration and Segregation in the Brain. Trends in Cognitive Sciences (2019) https:/doi.org/10.1016/j.tics.2019.04.002 (June 2, 2019).

25. W. Han, et al., A Neural Circuit for Gut-Induced Reward. Cell 175, 665-678.e23 (2018). 
-• An elegant study combining tracing and optogenetics demonstrating the link between gut sensory neurons and reward in mice

26. D. J. Levinthal, P. L. Strick, Multiple areas of the cerebral cortex influence the stomach. PNAS 117, 13078-13083 (2020).

- First anatomical tracing study revealing the brain regions controlling the stomach in rodents

27. G. Levakov, et al., Neural correlates of future weight loss reveal a possible role for brain-gastric interactions. Neuroimage 224, 117403 (2020).

- This fMRI study in humans suggests that weigh loss induces changes in the gastric network

28. M. Elam, P. Thorén, T. H. Svensson, Locus coeruleus neurons and sympathetic nerves: Activation by visceral afferents. Brain Research 375, 117-125 (1986).

29. T. Kukorelli, G. Juhász, Sleep induced by intestinal stimulation in cats. Physiol. Behav. 19, 355-358 (1977).

30. I. N. Pigarev, Neurons of visual cortex respond to visceral stimulation during slow wave sleep. Neuroscience 62, 1237-1243 (1994).

31. I. N. Pigarev, V. A. Bagaev, E. V. Levichkina, G. O. Fedorov, I. I. Busigina, Cortical visual areas process intestinal information during slow-wave sleep. Neurogastroenterol. Motil. 25, 268-275, e169 (2013).

32. C. Song, M. Boly, E. Tagliazucchi, H. Laufs, G. Tononi, BOLD signatures of sleep. bioRxiv, 531186 (2019).

33. H.-D. Park, O. Blanke, Coupling Inner and Outer Body for Self-Consciousness. Trends in Cognitive Sciences 23, 377-388 (2019).

34. V. Teckentrup, et al., Non-invasive stimulation of vagal afferents reduces gastric frequency. Brain Stimulation 13, 470-473 (2020).

35. G.-S. Hong, et al., Effect of transcutaneous vagus nerve stimulation on muscle activity in the gastrointestinal tract (transVaGa): a prospective clinical trial. Int J Colorectal Dis 34, 417-422 (2019).

36. I. Pigarev, et al., Visceral signals reach visual cortex during slow wave sleep: study in monkeys. Acta Neurobiologiae Experimentalis 66, 69-73 (2006).

37. A. Michaud, et al., Neuroanatomical changes in white and grey matter after sleeve gastrectomy. NeuroImage 213, 116696 (2020).

38. G. Li, et al., Bariatric surgery in obese patients reduced resting connectivity of brain regions involved with self-referential processing. Human Brain Mapping 39, 4755-4765 (2018).

39. F. Beyer, et al., Weight loss reduces head motion: Revisiting a major confound in neuroimaging. Human Brain Mapping 41, 2490-2494 (2020).

40. R. W. Mccallum, et al., Mechanisms of symptomatic improvement after gastric electrical stimulation in gastroparetic patients. Neurogastroenterology \& Motility 22, 161-e51 (2010).

41. X. Yu, et al., Antiemesis effect and brain fMRI response of gastric electrical stimulation with different parameters in dogs. Neurogastroenterology \& Motility 26, 1049-1056 (2014). 
42. C. Qin, Y. Sun, J. D. Z. Chen, R. D. Foreman, Gastric electrical stimulation modulates neuronal activity in nucleus tractus solitarii in rats. Autonomic Neuroscience 119, 1-8 (2005).

43. A. B. Allegra, A. A. Gharibans, G. E. Schamberg, D. C. Kunkel, T. P. Coleman, Bayesian inverse methods for spatiotemporal characterization of gastric electrical activity from cutaneous multielectrode recordings. PLOS ONE 14, e0220315 (2019).

44. T. Hashimoto, et al., Neural correlates of electrointestinography: Insular activity modulated by signals recorded from the abdominal surface. Neuroscience 289, 1-8 (2015).

45. A. A. Gharibans, et al., Artifact Rejection Methodology Enables Continuous, Noninvasive Measurement of Gastric Myoelectric Activity in Ambulatory Subjects. Scientific Reports 8, 5019 (2018). 